\title{
Especificidad Antropométrica y Nivel de Participación en Gimnasia Acrobática en Función del Sexo
}

\author{
Anthropometric Specificity and Level of Participation in Acrobatic Gymnastics Based on Sex
}

Yaiza Taboada-Iglesias ${ }^{1,4}$; Mercedes Vernetta-Santana²; Diego Alonso-Fernández ${ }^{3,4}$ \& Águeda Gutiérrez-Sánchez ${ }^{3,4}$

TABOADA-IGLESIAS, Y.; VERNETTA-SANTANA, M.; ALONSO-FERNÁNDEZ, D. \& GUTIÉRREZ-SÁNCHEZ, Á. Especificidad antropométrica y nivel de participación en gimnasia acrobática en función del sexo. Int. J. Morphol., 37(4):1534-1540, 2019.

RESUMEN: Las diferencias de sexo es un aspecto muy presente en el mundo laboral y deportivo. Esto se traduce en la diferenciación de actividades deportivas, determinadas como masculinas o femeninas. De ahí que el objetivo del estudio fue analizar el nivel de participación y características morfológicas en gimnastas de acrobática y ver las diferencias en relación al sexo y rol: portor y ágil. Se analizó la participación en los Campeonatos de España entre 2011 y 2018, y se comparó medidas antropométricas, composición corporal y de proporcionalidad, en función del rol de actuación y sexo. El Comité Autonómico de Ética de Investigación de la Xunta de Galicia (España) aprobó la metodología de trabajo. Los resultados mostraron un porcentaje mucho mayor de participación en función del sexo a favor del sexo femenino en todos los campeonatos analizados. Sin embargo, el peso, índice de masa corporal y el porcentaje muscular no fueron diferentes entre sexos en ninguno de los dos roles. De las 62 variables morfológicas evaluadas, existe un mayor predominio de las diferencias en los portores que en los ágiles en relación al sexo, destacando el porcentaje de grasa.

PALABRAS CLAVE: Gimnasia acrobática; Diferencias de sexo; Características morfológicas; Composición corporal.

\section{INTRODUCCIÓN}

Las diferencias físicas y capacitivas entre hombres y mujeres es un aspecto estudiado debido a la gran implicación social que conlleva. Desde el punto de vista fisiológico y de maduración sexual, las diferencias son bien establecidas, pero la discusión aparece en cuanto nos referimos a la función que son capaces de desempeñar.

La forma y tamaño corporal afecta en mayor medida que el sexo, en el desempeño de las funciones demandadas en las diferentes ocupaciones (Roberts et al., 2016).

Estas diferencias establecen de forma general que los hombres tienen unas mayores dimensiones en la talla y el peso, y menor masa grasa (Gordon et al., 1988; Malina \& Bouchard, 1991), y estableciéndose también una menor masa grasa en los miembros (Miller et al., 1993). Otros estudios también hacen referencia a la fuerza, estableciendo que las mujeres presentan menores niveles de fuerza en los miembros (Wilmore et al., 2008). Miller et al. sugieren que este nivel de fuerza menor, puede ser debido a la menor proporción del componente muscular. Así mismo, indicaron que las mujeres presentan una menor área transversal de los flexores de codo y extensores de rodilla, sin embargo no establecen diferencias de sexo en la relación de fuerza del área transversal.

En los niños y adolescentes también se han estudiado diferencias de sexo a nivel antropométrico y motriz. A nivel antropométrico, al igual que en los adultos, se encuentra que los niños pesan más que las niñas (Lazarevic et al., 2018). Del mismo modo, Quitério et al. (2017), encuentran diferencias en las habilidades motoras en niños de 6 años, encontrando que las niñas son más competentes en habilidades corporales, como el salto, mientras que los niños presentan más habilidad en las relativas a la manipulación de objetos. No obstante, no se establece si la forma, tamaño o proporción corporal está influyendo en estas variables o son debidas a otros factores.

\footnotetext{
${ }^{1}$ Facultad de Fisioterapia, Universidad de Vigo, España.

${ }^{2}$ Facultad de Ciencias del Deporte. Grupo de Investigación en Análisis y Evaluación de la Actividad Física y el Deporte (Cod.: CTS171) Universidad de Granada, España.

${ }^{3}$ Facultad de Ciencias de la Educación y el Deporte. Universidad de Vigo, España.

${ }^{4}$ Grupo de Investigación en Educación, Actividad Física y Salud (Gies10-DE3), Instituto de Investigación Sanitaria Galicia Sur (IIS Galicia Sur), SERGAS-UVIGO. Universidad de Vigo, España.
} 
En el ámbito deportivo también se ha estudiado la diferencia de sexo, desde puntos de vista biomecánicos (Bermejo Frutos et al., 2013), de condición física (SánchezAlcaraz Martínez et al., 2018), así como factores antropométricos y habilidades motrices (Di Cagno et al., 2009). Entre ellos, Bermejo Frutos et al. indicaron que las diferencias de sexo en el salto de altura, aumentan a medida que aumenta la edad de los deportistas.

Dentro de las disciplinas gimnásticas, Di Cagno et al. ya observaron que los hombres de Gimnasia Rítmica (GR) presentaban mayores dimensiones antropométricas que las mujeres de la misma especialidad, salvo en la talla sentado y la masa grasa que no tenían diferencia entre sexo. Sin embargo, sugieren que los deportistas de GR, en ambos sexos, deben tener características físicas similares para alcanzar los mejores resultados.

Las diferencias de sexo también se perciben desde el punto de vista de la participación, clasificando a los deportes por su carácter eminentemente femenino o masculino.

En el caso de las disciplinas gimnásticas, La Federación Internacional de Gimnasia (FIG) establece la participación de ambos sexos. La Gimnasia Artística dividida entre Gimnasia Artística Masculina (GAM) y Gimnasia Artística Femenina (GAF), la Gimnasia Trampolín con eventos de hombres y mujeres diferenciados, la Gimnasia Aeróbica en la que participan hombres y mujeres en diferentes categorías o en categorías mixtas, al igual que en la Gimnasia Acrobática (GA), en la que existen 5 tipos de agrupaciones de categoría: parejas femeninas, parejas masculinas y parejas mixtas, grupos de tres gimnastas mujeres y grupos de cuartetos hombres. La GR es la disciplina FIG con mayor problemática en la inclusión de hombres en las competiciones internacionales, no siendo reconocida actualmente por dicha institución, pero sí existen países actualmente con competiciones nacionales de GR masculina como en la Real Federación Española de Gimnasia.

La GA como ocurre con otros deportes gimnásticos como la Gimnasia Artística, la participación femenina es muy superior a la masculina (Kalinski, et al., 2018). Por este motivo, existe un gran número de estudios que se han centrado en comparar las características físicas y morfológicas de las practicantes de las diferentes disciplinas gimnásticas y no gimnásticas practicadas predominantemente por mujeres (Soric et al., 2008; Vicente-Rodríguez et al., 2007).

En GA también se diferencian dos roles, el de ágil y el de portor, con características funcionales muy diferenciadas. El portor es aquel gimnasta encargado de sostener al ágil, y el ágil el de realizar distintos elementos con o sin fase aérea propulsado por el portor (Vernetta-Santana et al., 2007).

Existen estudios que han comparado el perfil antropométrico entre gimnastas de GA, participantes en diferentes categorías de evento (grupos o parejas), que realizan el mismo rol, encontrando muy pocas diferencias (Taboada-Iglesias et al., 2017). Sin embargo, no existen estudios que establezcan si estas similitudes relativas al rol, se mantienen si las analizamos entre el mismo rol pero de diferente sexo.

Por tanto, este trabajo tiene como objetivo establecer las diferencias de sexo desde el punto de vista del nivel de participación deportiva y características morfológicas, y comprobar similitudes y diferencias en función del rol.

\section{MATERIAL Y MÉTODO}

Ética: Para la realización de este estudio se respetaron los procedimientos éticos marcados en la Ley Orgánica de Protección de Datos de Carácter Personal (Ley Orgánica 15/ 1999 de 13 de diciembre), siendo aprobado por el Comité Autonómico de Ética de Investigación de la Xunta de Galicia (España) (número de referencia 2015/672) y siguiendo los acuerdos de la declaración de Helsinki.

Participantes: La muestra fue compuesta por gimnastas españoles de GA, de nivel tanto nacional como internacional y escogidos por ser los componentes de mayor nivel de todo el territorio.

Se dividieron en función del rol y del sexo, para hacer un análisis diferenciado del grupo de ágiles [ágiles mujeres: $\mathrm{N}=54,11,23(3,04)$ años y ágiles hombres: $\mathrm{N}=4,12,30$ $(1,56)$ años] del de portores [Portoras mujeres: $\mathrm{N}=75,14,46$ $(2,09)$ años y portores hombres: $\mathrm{N}=17,15,09(4,49)$ años].

Procedimiento: Para el análisis de la participación en competición en función del sexo, se recogieron los informes de competición de la RFEG de los campeonatos de España de GA celebrados desde el año 2011 hasta el 2018.

Las variables morfológicas que se compararon entre sexo fueron diferentes medidas antropométricas, composición corporal, somatotipo y proporcionalidad.

Las 24 medidas antropométricas seleccionadas fueron tomadas siguiendo las recomendaciones de la ISAK (Marfell-Jones et al., 2006). Estas variables directas fueron 
peso $(\mathrm{P})$, talla $(\mathrm{T})$, talla sentado $(\mathrm{TS})$, envergadura $(\mathrm{E}), 5$ perímetros (perímetro del brazo relajado, brazo contraído, abdominal mínimo, glúteo y pierna máxima), 8 pliegues cutáneos (tríceps, bíceps, subescapular, supraespinal, cresta ilíaca, abdominal, muslo y pierna medial), 5 diámetros (biacromial, biileocrestal, capítulo-troclear del húmero, bicondíleo del fémur y biestiloideo de muñeca) y la longitud del miembro superior (LES). A partir de estas variables se calcularon el resto de los índices y variables. La longitud de las extremidades inferiores (LEI) se calculó restando la TS a la T.

La estrategia de De Rose y Guimarães (1980), basada en el método de cuatro componentes propuesto por Matiekga en 1921 fue la elegida para el cálculo de la composición corporal.

El somatotipo, encargado de describir la forma y tipo corporal, en tres componentes (endomorfia, mesomorfia y ectomorfia) fue calculado utilizando el método de HeathCarter (Carter \& Heath, 1990) y representado mediante la somatocarta.

Y por último, la proporcionalidad se estudió mediante dos metodologías. El Phantom de Ross \& Marfell-Jones (1991) en el que establecen un modelo de referencia unisex, en el cual se establecen los valores $\mathrm{Z}$ de proporcionalidad que son las variables absolutas transformadas relativas a la talla del sujeto, por lo que a partir de este método se establecerán 23 variables relativas a las medidas antropométricas tomadas y los índices de proporcionalidad, entre los que se encuentran el índice de masa corporal (IMC) (peso/estatura2), índice ponderal (IP) (IP= peso/talla3), la longitud relativa del miembro superior (LRES) $(\mathrm{LRES}=(\mathrm{LES} / \mathrm{T}) \mathrm{x} 100)$, la longitud relativa del miembro inferior (LREI) (LREI = (LEI/T)x100), el índice córmico $(\mathrm{IC}=$ (talla sentado/ talla)x 100), el índice esquelético (IE = [(talla - talla sentado)/ talla sentado]x100), el índice acromio-ilíaco (IAI= (diámetro ilíaco / diámetro acromial)x100) y la envergadura relativa $(\mathrm{ER}=($ envergadura $/$ talla $) \times 100)$.

Análisis estadístico: El análisis estadístico se realizó por un lado para el grupo de los ágiles y por otro para el de los portores con el SPSS 22.0 (Statistical Package for the Social Sciences). Se compararon entre hombres y mujeres las medias de cada variable. La prueba de Z de KolmogorovSmirnov comprobó la normalidad de la muestra y en el análisis comparativo se realizó la T de Student para comparar medidas independientes. Para la comparación de variables que no mantenían una distribución normal u homogénea se utilizó la prueba U de Mann-Whitney. El nivel de significación se situó en un valor de $\mathrm{p}<0,05$, para todos los test y pruebas realizadas.

\section{RESULTADOS}

En la Figura 1 se observa la diferencia de participación entre hombres y mujeres en los Campeonatos de España de GA. Existe una mayor participación femenina respecto a la masculina en todas las competiciones celebradas, así como un incremento en el número de participantes.

El análisis de los resultados morfológicos obtenidos, se establecerá primero para el grupo de los ágiles y en segundo lugar para el grupo de los portores.

\section{Ágiles}

Medidas antropométricas: De las 24 medidas antropométricas analizadas, tan solo 6 resultaron diferentes significativamente. Estas fueron la talla $(\mathrm{p}=, 043)$, la longitud de los miembros inferiores $(\mathrm{p}=, 009)$, el diámetro capítulotroclear del húmero $(\mathrm{p}=, 024)$, diámetro bicondíleo del fémur $(\mathrm{p}=, 007)$, el perímetro de brazo contraído $(\mathrm{p}=, 028)$ y el perímetro abdominal mínimo $(\mathrm{p}=, 025)$, siendo todas estas medidas superiores en los ágiles hombres. Sin embargo, ninguno de los pliegues obtuvo diferencias significativas (Fig. 2).

Composición corporal: En cuanto la composición corporal, se encontraron diferencias significativas en el \% de gra$\mathrm{sa}(\mathrm{p}=, 004)$ y en el \% residual $(\mathrm{p}=, 001)$. Este último debido a la propia definición de la variable. El \% de grasa obtuvo valores mayores en las mujeres que en los hombres.

Somatotipo: Los diferentes componentes del somatotipo no establecieron diferencias significativas entre ellos (endomorfia $\mathrm{p}=, 286$; mesomorfia $\mathrm{p}=, 715$; ectomorfia $\mathrm{p}=, 502)$. Así mismo, tanto el somatotipo de los ágiles hombres como de las ágiles mujeres se define como ectomesomorfo, ya que la mesomorfia es dominante seguida de la ectomorfia. En la Figura 3, se observa cómo se comporta el somatotipo de ambos sexos.

Phantom de proporcionalidad: Del análisis de proporcionalidad basado en el método Phantom, se establecen diferencias significativas en sólo dos de las 23 variables $Z$ de proporcionalidad. Estas diferencias se encontraron en la $\mathrm{Z}$ Talla sentado $(\mathrm{p}=, 032)$ y en la $\mathrm{Z}$ longitud de los miembros inferiores $(\mathrm{p}=, 006)$. En ambos casos, la proporcionalidad indica que se trata de variables más pequeñas que la media del sujeto. Sin embargo, proporcionalmente la TS de los hombres es más pequeña que la de las mujeres y la LEI más pequeña en las mujeres. El resto de las variables obtuvieron valores negativos, salvo los valores $\mathrm{Z}$ de los diámetros bicondíleo del fémur, biestiloideo de muñeca y biepicondíleo del húmero. 


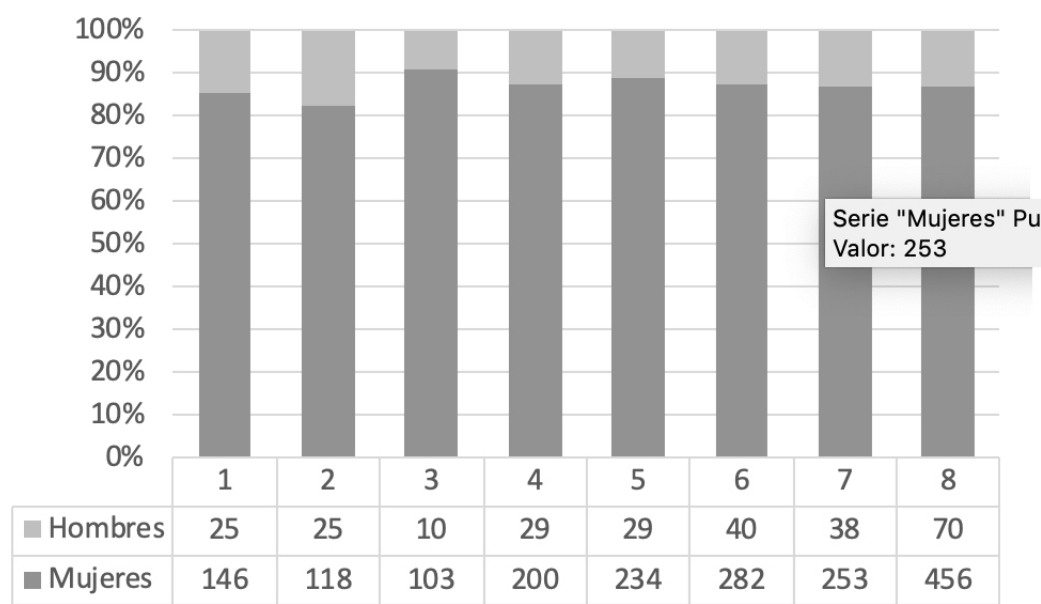

Fig. 1. Distribución de la participación por sexos en los últimos 7 campeonatos de España de Gimnasia Acrobática.

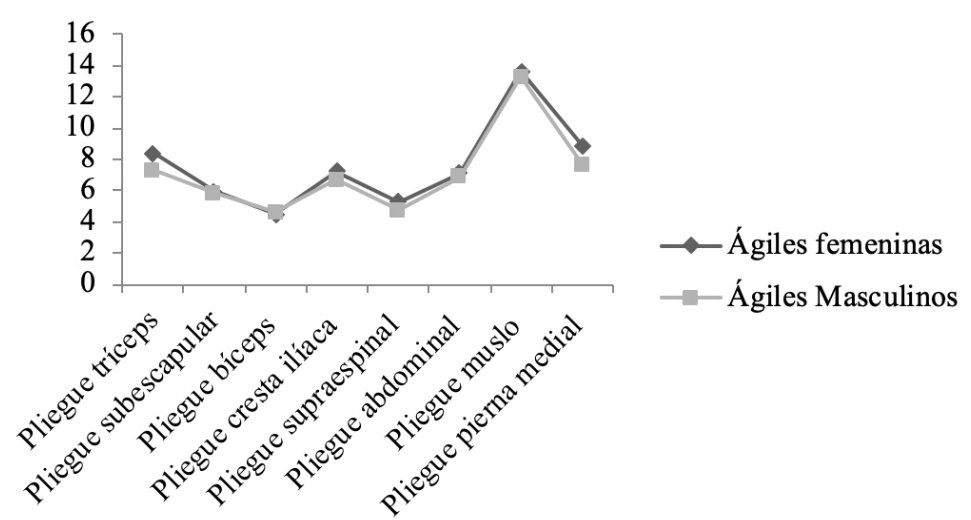

Fig. 2. Distribución de los pliegues cutáneos de los ágiles de diferente sexo.

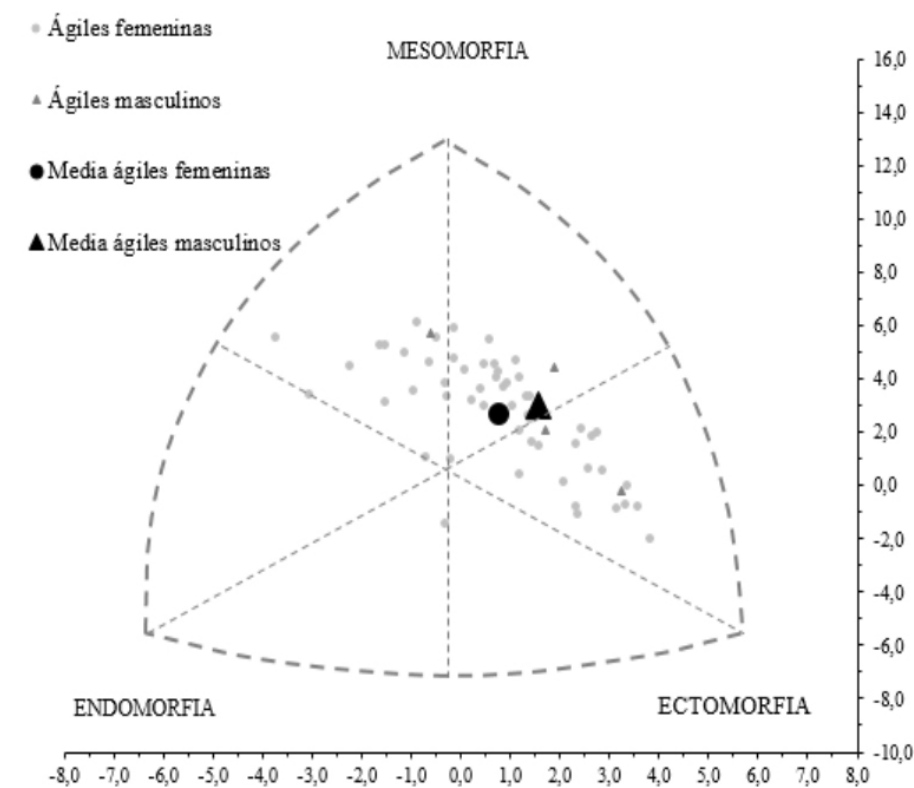

Fig. 3. Somatocarta de los valores medios e individuales en los diferentes sexos de ágiles.
Índices de proporcionalidad: En cuanto a los índices de proporcionalidad, no se encontraron diferencias significativas en el IMC ( $\mathrm{p}=, 382)$. Del resto de los índices, tan solo se presentaron diferencias en 3 de ellos. En la LREI $(\mathrm{p}=, 007)$ clasificándose como braquiesquelético o de miembros inferiores cortas tanto en los ágiles hombres como en las ágiles mujeres, pese a ser significativamente diferentes. En el IE $(\mathrm{p}=, 006)$, también establece que los ágiles masculinos y femeninos presentan los miembros inferiores cortas o braquiesqueléticos. Y el IC $(p=, 007)$ presenta un tronco corto los ágiles hombres y un troco intermedio las mujeres.

\section{Portores}

Medidas antropométricas: De las 24 variables analizadas, solo 8 resultaron ser significativamente diferentes. Estas fueron los diámetros capítulo-troclear del húmero $(\mathrm{p}=, 000)$, el biestiloideo de muñeca $(\mathrm{p}=, 000)$ y el bicondíleo del fémur $(\mathrm{p}=, 000)$, el perímetro abdominal mínimo $(\mathrm{p}=, 021)$ y los pliegues del tríceps $(\mathrm{p}=, 001)$, supraespinal $(\mathrm{p}=, 026)$, abdominal $(\mathrm{p}=, 035)$ y el del muslo $(\mathrm{p}=, 000)$. Estas diferencias en los pliegues y su distribución se pueden observar en la Figura 4.

El \% de grasa, el \% óseo y el \% residual obtuvieron diferencias significativas entre sexos. Las diferencias en el \% residual se derivan de la propia definición de la variable. Las diferencias en el \% de grasa $(p=, 000)$ indican mayores valores en las mujeres, y las diferencias en el \% óseo $(p=, 009)$ muestran valores superiores en los hombres. Sin embargo, el \% muscular no obtuvo diferencias significativas entre ambos grupos.

Somatotipo: El somatotipo de los portores establece diferencias en la endomorfia $(\mathrm{p}=, 031)$ y en la mesomorfia $(\mathrm{p}=, 000)$. Siendo la endomorfia mayor en las mujeres y la mesomorfia mayor en los hombres. Se define como mesomorfo balanceado en hombres, ya que la mesomorfia es dominante y los otros dos componentes no se diferencian en más de media unidad, y central en muje- 
res, ya que ningún componente se diferencia en más de una unidad. En la Figura 5 se puede ver cómo se comportan los somatotipos de ambos sexos representados por la somatocarta.

Phantom de proporcionalidad: De las 23 variables de proporcionalidad, 12 obtuvieron diferencias significativas entre sexos. Entre ellas, las $\mathrm{Z}$ de los diámetros capítulo troclear del húmero $(\mathrm{p}=, 000)$, biestiloideo de muñeca $(\mathrm{p}=, 000)$ y bicondíleo del fémur $(\mathrm{p}=, 000)$, las $\mathrm{Z}$ de los perímetros abdominal mínimo $(\mathrm{p}=, 000)$, glúteo $(\mathrm{p}=, 025)$, brazo relajado $(\mathrm{p}=, 041)$ y brazo contraído $(\mathrm{p}=, 017)$ y las $\mathrm{Z}$ de los pliegues del tríceps $(\mathrm{p}=, 002)$, cresta ilíaca $(\mathrm{p}=, 047)$, muslo anterior $(\mathrm{p}=, 013)$ y pierna medial $(\mathrm{p}=, 002)$.

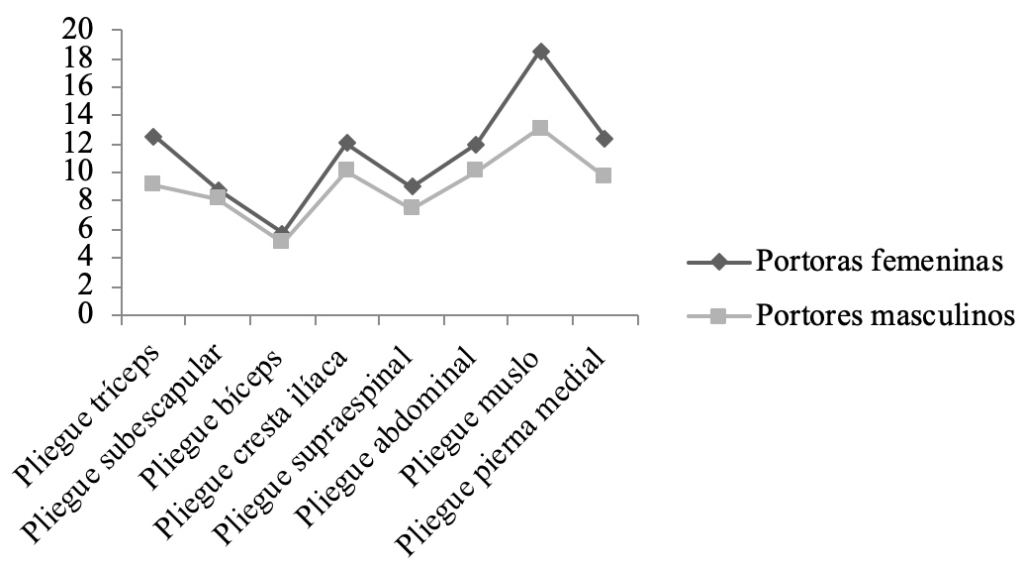

Fig. 4. Distribución de los pliegues cutáneos de los portores de diferente género.

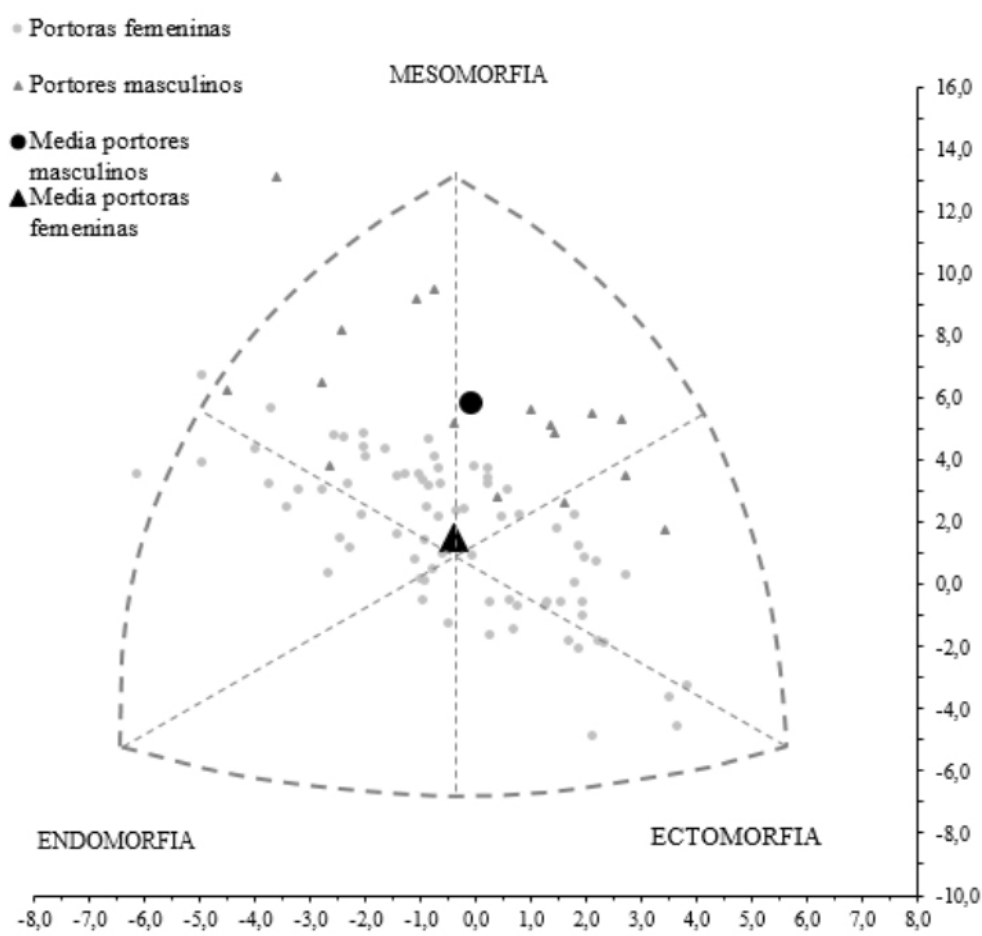

Fig. 5. Somatocarta de los valores medios e individuales en los diferentes sexos de portores
Índices de proporcionalidad: Pese a las diferencias en la proporcionalidad mediante el Phantom, tan solo el IAI resultó ser diferente significativamente $(\mathrm{p}=, 022)$ entre portores hombres y portoras mujeres de entre todos los índices de proporcionalidad analizados. Las diferencias en este índice muestran un tronco trapezoidal en los hombres e intermedio en las mujeres. Cabe destacar que no se encontraron diferencias tampoco en el $\operatorname{IMC}(\mathrm{p}=, 325)$.

\section{DISCUSIÓN}

La mayor participación de mujeres en GA es por el momento muy destacable. Se observa una participación femenina muy superior a la masculina al igual que en otras disciplinas gimnásticas, como resaltaron Kalinski et al. en Gimnasia Artística, aun siendo ésta dividida en GAF y GAM. Se afirma que en GA la participación masculina es escasa. Esta diferenciación es mayor incluso en GR, en la que aún no se reconoce la participación masculina en la FIG, siendo en 2009, España pionera a nivel mundial al organizar por primera vez un campeonato nacional masculino en esta disciplina.

En nuestro estudio, se observa que a pesar de la transición de ocho años desde el primer campeonato analizado en 2011 hasta el último 2018, y el aumento en la participación total de la muestra (de 171 en 2011 a 526 en 2018), el porcentaje de participación de los hombres es bajo con una X de 12,92 $\%$ en relación al de las mujeres con una $\mathrm{X}$ de 87,25\% teniendo en cuenta los campeonatos observados. Esto justificaría la necesidad de crear un perfil exclusivamente femenino.

Las escasas diferencias encontradas entre grupos en relación al sexo, da respuesta al predominio de la función frente al sexo. En nuestro estudio se confirma que sujetos con unas dimensiones corporales similares son capaces de realizar las mismas tareas o trabajo físico, independientemente de ser hombres o mujeres, datos que corroboran lo indicado por (Roberts et al.). 
El peso y la talla son las variables que con mayor frecuencia difieren, estableciéndose que los hombres miden y pesan más que las mujeres (Gordon et al.; Malina \& Bouchard) e incluso que los niños pesan más que las niñas (Lazarevic et al.). Sin embargo, estas variables no presentan diferencias entre nuestros sujetos, diferenciándose tan solo la talla en el grupo de los ágiles.

Desde el punto de vista de la composición corporal, si se encuentran diferencias entre grupos. Como ya se apunta en la población general, los hombres presentan menor masa grasa (Gordon et al.; Malina \& Bouchard), al igual que pasa con los gimnastas analizados. Aunque Miller et al. indicaban una menor masa grasa en los miembros en los hombres que en las mujeres, la distribución de pliegues en los ágiles, no muestra diferencias significativas en ellas. Sin embargo, esta diferencia si se encuentra en los portores, son diferencias significativas en los pliegues del tríceps y del muslo. Por el contrario, el porcentaje muscular no obtiene diferencia de sexo, que podría ser indicativo del menor nivel de fuerza que establece Miller et al. en las mujeres. Pero estos autores lo señalan a una menor proporción del componente muscular.

No obstante, pese a encontrar pocas diferencias entre sexos, sí se observa la tendencia apuntada por Bermejo Frutos et al. en parámetros biomecánicos del salto de altura, de que las diferencias entre sexos aumentan con la edad, ya que el grupo de portores, con una media de edad superior, presenta mayor número de variables con diferencias significativas que el de los ágiles. Sin embargo, que los ágiles presenten más similitud que los portores, también puede deberse a la mayor especificidad del rol del ágil, encargado de realizar elementos muy variados sostenidos y propulsados por los portores.

Las diferencias encontradas en los gimnastas de GR de Di Cagno et al. en la talla y LEI, tan sólo se asemejan a las encontradas entre los ágiles, ya que entre los portores no presentan diferencias significativas entre sexos. Además, las diferencias de sexo encontradas en GR en el peso y el IMC, no se confirman en nuestros gimnastas de GA, ni en el grupo de ágiles ni en el de portores. Por otro lado, en lo relativo a la masa grasa, ni los gimnastas de GR (Di Cagno et al.) ni los de GA, presentan diferencias entre hombres y mujeres.

Aunque no hay estudios que comparen directamente a hombres y mujeres de otras disciplinas gimnásticas, sí existen referencias de cómo es la morfología característica de éstas. Es el caso de la gimnasia Trampolín en la que ambos sexos presentan similitudes en sus miembros superiores cortos, miembros inferiores largos, un IC que muestra un tronco de tamaño intermedio y un IAI con forma trapezoidal (Gómez-Landero, 2010). Estas similitudes en la interpreta- ción de los índices relativos a la longitud de los miembros inferiores, también la encontramos en nuestros gimnastas, sin embargo, en los ágiles, aunque se clasifican como miembros inferiores cortos en ambos sexos, tanto el IE como la LREI presentan diferencias significativas. El IAI, resultó igual en los ágiles como en los gimnastas de Trampolín, Sin embargo, en los portores sí hubo diferencias, mostrando un tronco trapezoidal en los hombres y medio en las mujeres.

En Gimnasia Artística, Smoleuskiy \& Gaverdouskiy (1996), ya establecieron que tanto hombres como mujeres se caracterizaban por tener los hombros anchos. Por ello, en relación al IAI, que muestra la relación entre la anchura de hombros y cadera, podemos afirmar que en los ágiles se cumple este parecido entre sexos, pero no en los portores, ya que los hombres presentan unos hombros más anchos, en relación a la cadera, que las mujeres.

En cuanto al somatotipo, solo se encontraron diferencias significativas en los portores. La mesomorfia de los portores es mayor en los hombres que en las mujeres. Este mayor predominio se asemeja a los resultados encontrados en Gimnasia Artística, en la que los gimnastas de GAM presentan generalmente un somatotipo mesomorfo balanceado (Ferreira João \& Fernandes Filho, 2015), mientras que en GAF el componente ectomórfico cobra mayor importancia. Siendo el somatotipo en GAF definido como ectomorfo mesomorfo por Massidda et al. (2013) y ecto-mesomorfo por Irurtia Amigó et al. (2009).

Debido a encontrar en los portores más diferencias que en los ágiles, la edad puede ser un factor condicionante. No obstante, este hecho puede ser provocado por una mayor especificidad del rol.

Las limitaciones del estudio son fundamentalmente la poca muestra masculina, derivada de la poca participación de este sexo, y la diferencia de edad entre ágiles y portores que no permite comparar si el mayor número de diferencias entre los portores es debido a la especificidad del rol o de la edad.

Como líneas de investigación futuras podrían proponerse estudios de regresión y comparativos entre el éxito deportivo o habilidad motriz con las variables antropométricas y el sexo, estableciendo si existe relación entre ellas y cuál tiene más peso. También sería interesante, establecer las diferencias de sexo entre las diferentes categorías de edad, de cara a obtener resultados más consistentes sobre la influencia de la edad.

AGRADECIMIENTOS. A todos los gimnastas de acrobática y entrenadores de los clubs de Gimnasia en España. 
TABOADA-IGLESIAS, Y.; VERNETTA-SANTANA, M.; ALONSO-FERNÁNDEZ, D. \& GUTIÉRREZ-SÁNCHEZ, Á. Anthropometric specificity and level of participation in acrobatic gymnastics based on sex. Int. J. Morphol., 37(4):1534-1540, 2019.

SUMMARY: Sex differences are a very present aspect in the world of work and sports. This translates into the differentiation of sports activities, determined as masculine or feminine. Hence, the aim of the study was to analyze the level of participation and morphological characteristics in acrobatic gymnasts and to see the differences in relation to sex and role: Base and top. Participation in the Spanish Championships between 2011 and 2018 was analyzed, and anthropometric measures, body composition and proportionality were compared, depending on the role of performance and sex. The Autonomous Committee of Research Ethics of the Xunta de Galicia (Spain) approved the work methodology. The results showed a much higher percentage of participation according to sex in favor of the female sex in all the analyzed championships. However, the weight, body mass index and muscle percentage were not different between sexes in either of the two roles. Of the 62 morphological variables evaluated, there is a greater predominance of differences in the bases than in the tops ones in relation to sex, highlighting the percentage of fat.

KEY WORDS: Acrobatic gymnastics; Sex differences; Morphological characteristics; Body composition.

\section{REFERENCIAS BIBLIOGRÁFICAS}

Bermejo Frutos, J.; López Elvira, J. L. \& Palao Andrés, J. M. Diferencias de género en salto de altura según categorías de edad. Apunts Educ. Fis. Deportes, (111):62-9, 2013.

Carter, J. E. L. \& Heath, H. B. Somatotyping: development and applications. Cambridge (UK), Cambridge University Press, 1990.

De Rose, E. \& Guimarães, A. C. A. Model for Optimization of Somatotype in Young Athletes. En: Ostyn, M.; Beunen, G. \& Simons, J. Kinanthropometry II. Baltimore, University Park Press, 1980.

Di Cagno, A.; Baldari, C.; Battaglia, C.; Monteiro, M. D.; Pappalardo, A.; Piazza, M. \& Guidetti, L. Factors influencing performance of competitive and amateur rhythmic gymnastics--gender differences. $J$. Sci. Med. Sport, 12(3):411-6, 2009.

Ferreira João, A. \& Fernandes Filho, J. A. Somatotype and body composition of elite brazilian gymnasts. Sci. Gymnast. J., 7(2):45-54, 2015.

Gómez-Landero, A. Análisis del Perfil Motor, Morfológico y Funcional del Trampolín como Deporte Gimnástico. Tesis de Doctorado. Granada, Universidad de Granada, 2010.

Gordon, C.; Churchill, T.; Clauser, C.; Bradtmiller, B.; McConville, J.; Tebbetts, I. \& Walker, R. Anthropometric Survey of U.S. Army Personnel: Methods and Summary Statistics. Natick, Army Natick Research, Development and Engineering Center, 1988.

Irurtia Amigó, A.; Busquets Faciabén, A.; Marina Evrard, M.; Galilea Ballarini, P. A. \& Carrasco Marginet, M. Talla, peso, somatotipo y composición corporal en gimnastas de elite españoles desde la infancia hasta la edad adulta. Apunts Med. Esport, 44(161):18-28, 2009.

Kalinski, S. D.; Atikovic, A. \& Jelaska I. Gender differences in consecutive participation in artistic gymnastics at the Olympic Games from 1996 to 2016. Sci. Gymnast. J., 10(1):51-67, 2018.

Lazarevic, P.; Milosavljevic, S.; Lazarevic, S.; Markovic, V. \& Savic, A. Different levels of motor abilities in boys and girls aged 10 and 9. Facta Univ. Phys. Educ. Sport, 16(1):189-99, 2018.
Malina, R. M. \& Bouchard, C. Growth, Maturation and Physical Activity. Champaign, Human Kinetics Academics, 1991.

Marfell-Jones, M.; Olds, T.; Stewart, A. \& Carter, L. International Standards for Anthropometric Assessment. Potchefstroom, ISAK, 2006.

Massidda, M.; Toselli, S.; Brasili, P. \& Calò, C. M. Somatotype of elite Italian gymnasts. Coll. Antropol., 37(3):853-7, 2013.

Miller, A. E.; MacDougall, J. D.; Tarnopolsky, M. A. \& Sale, D. G. Gender differences in strength and muscle fiber characteristics. Eur. J. Appl. Physiol. Occup. Physiol., 66(3):254-62, 1993.

Quitério, A. Q.; Costa, J.; Martins, M.; Martins, J.; Onofre, M.; Gerlach, E.; Sheuer, C. \& Herrmann, C. Educação Física: Avaliação das competências motoras em alunos de seis anos, do primeiro ano de escolaridade. Retos, (31):259-63, 2017.

Roberts, D.; Gebhardt, D. L.; Gaskill, S. E.; Roy, T. C. \& Sharp, M. A. Current considerations related to physiological differences between the sexes and physical employment standards. Appl. Physiol. Nutr. Metab., 41(6 Suppl. 2):S108-20, 2016.

Ross, W. D. \& Marfell-Jones, M. J. Kinanthropometry. In: MacDougall, J. D. (Ed.), Physiological Testing of the High-Performance Athlete. $2^{\text {nd }}$ ed. Ottawa, Canadian Association of Sports Sciences, Sports Medicine Council of Canada, 1991.

Sánchez-Alcaraz Martínez, B. J.; Orozco Ballesta, V.; Courel Ibáñez, J. \& Sánchez Pay, A. Evaluación de la velocidad, agilidad y fuerza en jóvenes jugadores de pádel. Retos, 34:263-6, 2018.

Smoleuskiy, V. \& Gaverdouskiy, I. Tratado General de Gimnasia Artística Deportiva. Barcelona, Paidotribo, 1996.

Soric, M.; Misigoj-Durakovic, M. \& Pedisic, Z. Dietary intake and body composition of prepubescent female aesthetic athletes. Int. J. Sport Nutr. Exerc. Metab., 18(3):343-54, 2008.

Taboada-Iglesias, Y.; Santana, M. V. \& Gutiérrez-Sánchez, A. Anthropometric profile in different event categories of acrobatic gymnastics. J. Hum. Kinet., 57:169-79, 2017.

Vernetta-Santana, M.; Rodríguez, J. J. \& Bedoya, J. L. La utilización del registro de los tiempos de intervención de las acciones motrices en la gimnasia acrobática. Lecturas: EF y deportes. Efdeportes Rev. Dig., 12(110):1-11, 2007. Disponible en: https://www.efdeportes.com/efd110/ acciones-motrices-en-la-gimnasia-acrobatica.htm

Vicente-Rodríguez, G.; Dorado, C.; Ara, I.; Perez-Gomez, J.; Olmedillas, H.; Delgado-Guerra, S. \& Calbet, J. A. Artistic versus rhythmic gymnastics: effects on bone and muscle mass in young girls. Int. J. Sports Med., 28(5):386-93, 2007.

Wilmore, J. H.; Costill, D. L. \& Kenney, W. L. Physiology of Sport and Exercise. 4th ed. Champaign, Human Kinetics, 2008.

Dirección de correspondencia:

Águeda Gutiérrez-Sánchez

Universidad de Vigo

Facultad de Ciencias de la Educación y el Deporte

Campus A Xunqueira, s/n. 36005

ESPAÑA

Email: agyra@uvigo.es

Recibido : 09-05-2019

Aceptado: 01-07-2019 